$09 ; 12$

\title{
Принцип подобия траекторий при движении заряженных частиц с разными массами в однородных по Эйлеру электрических и магнитных полях
}

\author{
(C) И.А. Аверин ${ }^{1,2}$, А.С. Бердников ${ }^{1, \uparrow}$, Н.Р. Галль ${ }^{1,3}$ \\ ${ }_{1}^{1}$ Институт аналитического приборостроения РАН, Санкт-Петербург \\ ${ }^{2}$ Санкт-Петербургский политехнический университет им. Петра Великого, \\ Санкт-Петербург \\ ${ }^{3}$ Физико-технический институт им. А.Ф. Иоффе РАН, Санкт-Петербург \\ ฯ E-mail: asberd@yandex.ru
}

Поступило в Редакцию 12 июля 2016 г.

Электрические поля, которые описываются однородными по Эйлеру потенциалами, и подчиняются принципу подобия траекторий, сформулированному Ю.К. Голиковым, являются полезным инструментом для разработки электроннооптических и ионно-оптических систем с уникальными свойствами. В этой работе принцип подобия траекторий обобщается на случай магнитных полей и комбинированных электрических и магнитных полей, однородных по Эйлеру.

DOI: 10.21883/PJTF.2017.03.44225.16402

Энерго- и масс-анализаторы, способные охватить большой диапазон энергий или массовых чисел за одно измерение (спектрографы), это важный и относительно слабо изученный класс электрофизических приборов [1]. Именно на пути перехода от сканирующей моды, традиционно используемой в электронной спектроскопии и масс-спектрометрии к одновременной регистрации всех частиц в требуемом диапазоне измеряемого параметра (энергии или массового числа) видится будущее этих областей экспериментальной физики.

Оптика систем, обладающих хорошими спектрографическими свойствами, сильно отличается от оптики систем с хорошими спектрометрическими свойствами, а разработка таких систем приводит к совершенно новым оптическим задачам и соответственно к новым способам их решения. Здесь полезным инструментом являются поля, 
однородные по Эйлеру: электрические, удовлетворяющие тождеству $\mathbf{E}(\lambda x, \lambda y, \lambda z)=\lambda^{n} \mathbf{E}(x, y, z)$, либо магнитные, удовлетворяющие тождеству $\mathbf{B}(\lambda x, \lambda y, \lambda z)=\lambda^{n} \mathbf{B}(x, y, z)$, где $n-$ фиксированный порядок однородности, $\lambda-$ произвольный множитель. Этим электрическим полям, преимущественно с целочисленными порядками однородности, посвящено достаточно много публикаций [2-4], принадлежащих проф. Ю.К. Голикову и последователям его школы оптики заряженных частиц, по большей части с упором на спектрографические системы для нужд электронной спектроскопии. Задачей данной работы является обобщение предложенного Ю.К. Голиковым принципа подобия траекторий в электрических полях, однородных по Эйлеру, на однородные по Эйлеру магнитные поля и на комбинированные однородные электрические и магнитные поля, в том числе и с нецелочисленными порядками однородности. Тем самым становится возможным использовать при разработке спектрографических корпускулярно-оптических систем новые классы электрических и магнитных полей, что значительно расширяет свободу синтеза и оптимизации таких устройств.

Пусть заданы электрическое поле $\mathbf{E}(\mathbf{r})$ и магнитное поле $\mathbf{B}(\mathbf{r})$, где $\mathbf{r}=(x, y, z)$ - декартовы координаты в трехмерном пространстве. Уравнения движения заряженных частиц в таком комбинированном поле имеют вид

$$
\ddot{\mathbf{r}}(t)=\frac{e}{m} \mathbf{E}(\mathbf{r}(t))+\frac{e}{m c}[\dot{\mathbf{r}}(t) \times \mathbf{B}(\mathbf{r}(t))],
$$

где $\mathbf{r}(t)=(x(t), y(t), z(t))$ - меняющиеся во времени координаты частицы, $t-$ время, $\mathbf{E}(\mathbf{r})-$ напряженность электрического поля, $\mathbf{B}(\mathbf{r})$ - индукция магнитного поля, $c-$ множитель, зависящий от выбранной системы единиц, $m-$ масса частицы, $e-$ заряд частицы. Далее будем считать заряд частиц постоянным и использовать термин „масса“ в значении „массовое число“.

Пусть $\mathbf{E}(\mathbf{r})$ и $\mathbf{B}(\mathbf{r})$ - однородные по Эйлеру функции порядка $p$ и $q$, так что $\mathbf{E}(\lambda \mathbf{r}) \equiv \lambda^{p} \mathbf{E}(\mathbf{r}), \mathbf{B}(\lambda \mathbf{r}) \equiv \lambda^{q} \mathbf{B}(\mathbf{r})$, а $\mathbf{r}(t)$ - решение уравнения (1). Может ли масштабированная функция $\boldsymbol{\rho}(t)=\lambda \mathbf{r}(\varepsilon t)$ быть решением уравнения (1) для частиц с массами $m^{\prime}=\mu m$ ? Да, если $\lambda, \varepsilon, \mu$ выбраны так, что $\lambda \varepsilon^{2}=\lambda^{p} / \mu=\lambda^{q+1} \varepsilon / \mu$. Действительно, так как $d \boldsymbol{\rho} / d t=\lambda \varepsilon d \mathbf{r} / d t$

Письма в ЖТФ, 2017, том 43, вып. 3 


$$
\begin{aligned}
& \text { и } d^{2} \boldsymbol{\rho} / d t^{2}=\lambda \varepsilon^{2} d^{2} \mathbf{r} / d t^{2}, \text { то } \\
& \ddot{\boldsymbol{\rho}}-\frac{e}{\mu m} \mathbf{E}(\boldsymbol{\rho})-\frac{e}{\mu m c}[\dot{\boldsymbol{\rho}} \times \mathbf{B}(\boldsymbol{\rho})]=\lambda \varepsilon^{2} \ddot{\mathbf{r}}-\frac{e}{\mu m} \mathbf{E}(\lambda \mathbf{r})-\frac{e}{\mu m c}[\lambda \varepsilon \dot{\mathbf{r}} \times \mathbf{B}(\lambda \mathbf{r})] \\
& =\lambda \varepsilon^{2} \ddot{\mathbf{r}}-\frac{\lambda^{p}}{\mu} \frac{e}{m} \mathbf{E}(\mathbf{r})-\frac{\lambda^{q+1} \varepsilon}{\mu} \frac{e}{m c}[\mathbf{r} \times \mathbf{B}(\mathbf{r})] \\
& =\lambda \varepsilon^{2}\left(\ddot{\mathbf{r}}-\frac{e}{m} \mathbf{E}(\mathbf{r})-\frac{e}{m c}[\mathbf{r} \times \mathbf{B}(\mathbf{r})]\right) .
\end{aligned}
$$

Тем самым функции $\boldsymbol{\rho}(t)$ и $\mathbf{r}(t)$ одновременно оказываются решениями соответствующих уравнений движения. Случаи $\mathbf{B}=0$ и $\mathbf{E}=0$ разбираются аналогично.

Рассмотрим частные варианты.

1. Электрическое поле $(\mathbf{B}=0)$. Условия на $\lambda, \varepsilon, \mu$ сводятся к $\lambda \varepsilon^{2}=\lambda^{p} / \mu$, т.е. к выбору $\varepsilon=\lambda^{(p-1) / 2} \mu^{-1 / 2}$. Это соответствует масштабированию начальной кинетической энергии для траектории $\boldsymbol{\rho}(t)$ в $\gamma=\mu \lambda^{2} \varepsilon^{2}=\lambda^{p+1}$ раз (независимо от масштаба массы), причем масштабирование по энергии $\gamma$ можно рассматривать как независимый параметр, а масштабирование по времени $\varepsilon-$ как функцию от масштабирования по энергии и по массе. Начальные координаты траектории $\boldsymbol{\rho}(t)$ масштабируются в $\lambda$ раз, начальные углы остаются прежними. Значит, если масштабировать начальные координаты в $\lambda$ раз, начальную кинетическую энергию в $\lambda^{p+1}$ раз и сохранить углы вылета неизменными, то при любых $\lambda, \mu$ новая траектория будет масштабированной в $\lambda$ раз версией исходной траектории. При этом $\mu$ определяет масштабирование по времени, т. е. скорость движения вдоль траектории, но не форму траектории. Это свойство траекторий в электрических полях, однородных по Эйлеру, было названо Ю.К. Голиковым принципом подобия траекторий [2,3] и впоследствии было им обобщено и на другие классы электрических полей [5,6]. Данное свойство позволяет использовать электрические поля, однородные по Эйлеру, для электронных спектрографов [2-4].

2. Магнитное поле $(\mathbf{E}=0)$. Условия на $\lambda, \varepsilon, \mu$ сводятся к $\lambda \varepsilon^{2}=\lambda^{q+1} \varepsilon / \mu$, т.е. к выбору $\varepsilon=\lambda^{q} \mu^{-1}$. В таком случае у траектории $\boldsymbol{\rho}(t)$ модуль начального импульса масштабируется в $\chi=\mu \lambda \varepsilon=\lambda^{q+1}$ раз (независимо от масштаба массы). Масштабирование по модулю импульса $\chi$ может рассматриваться как независимый параметр, а

Письма в ЖТФ, 2017, том 43, вып. 3 
масштабирование по времени $\varepsilon-$ как функция от масштабирования модуля импульса и массы. Начальные координаты масштабируются в $\lambda$ раз, начальные углы остаются прежними. Значит, если масштабировать начальные координаты частицы в $\lambda$ раз, начальный импульс (точнее, модуль импульса) в $\lambda^{q+1}$ раз ${ }^{1}$ и сохранить неизменными углы вылета, то при любых $\lambda, \mu$ новая траектория будет масштабированной в $\lambda$ раз версией исходной траектории. При этом $\mu$ определяет масштабирование по времени, т.е. скорость движения вдоль траектории, но не форму траектории. Этот результат обобщает принцип подобия траекторий на магнитные поля, однородные по Эйлеру, и позволяет использовать такие магнитные поля как для чисто магнитных электронных спектрографов [1] и масс-спектрографов [7], так и для статических массанализаторов спектрографического типа с двойной фокусировкой $[8,9]$. (Эти применения хорошо известны для магнитного поля $\mathbf{B}=$ const частного случая поля, однородного по Эйлеру.)

3. Комбинированные поля. Условие $\lambda \varepsilon^{2}=\lambda^{p} / \mu=\lambda^{q+1} \varepsilon / \mu$ означает, что для масштабированной в $\lambda$ раз траектории следует выбирать $\varepsilon=\lambda^{p-q-1}, \mu=\lambda^{2 q-p+1}$. Тогда начальная кинетическая энергия, равно как и полная начальная энергия, масштабируются в $\gamma=\mu \lambda^{2} \varepsilon^{2}=\lambda^{p+1}$ раз, а модуль начального импульса масштабируется в $\chi=\mu \lambda \varepsilon=\lambda^{q+1}$ раз. Начальные координаты масштабируются в $\lambda$ раз, начальные углы остаются прежними. В результате при масштабировании начальных координат в $\lambda$ раз, начальной кинетической или полной энергии в $\lambda^{p+1}$ раз (либо начального импульса в $\lambda^{q+1}$ раз), массы в $\lambda^{2 q-p+1}$ раз и сохранении прежних начальных углов траектория будет масштабированной в $\lambda$ раз по сравнению с исходной версией. Это является обобщением принципа подобия траекторий для случая комбинирования (наложения) полей, однородных по Эйлеру.

Рассмотрим частные (вырожденные) случаи наложения полей. Для траекторий с одной и той же массой (случай $\mu=1, p=2 q+1$ ) траектории с энергией, масштабированной в $\gamma$ раз, будут геометрически масштабированы в $\gamma^{1 /(p+1)}$ раз, если порядки однородности электрического и магнитного поля согласованы как $p=2 q+1$. Для траекторий с одной и той же энергией (случай $\gamma=1, p=-1)$ частицы с массами, масштабированными в $\mu$ раз, будут двигаться по траекториям,

\footnotetext{
${ }^{1}$ Для случая постоянной массы ( $\mu=1$, электронный пучок) масштабирование модуля импульса в $\chi=\lambda^{q+1}$ раз и масштабирование кинетической энергии в $\gamma=\lambda^{2 q+2}$ раз - это одно и то же.
} 
масштабированным в $\mu^{1 /(2 q+2)}$, если электрическое поле выбрано как $p=-1$. Для траекторий частиц одного и того же импульса (случай $\chi=1, q=-1)$ частицы с массами, масштабированными в $\mu$ раз, будут двигаться по траекториям, масштабированным в $\mu^{-1 /(p+1)}$ раз, если магнитное поле выбрано как $q=-1$. Наконец, при $p=-1, q=-1$ параллельный пучок с фиксированными углами, энергиями и массами, входящий в комбинированное поле, преобразуется в выходной параллельный пучок, причем угол поворота (а также коэффициент сжатия) является функцией входного угла, энергии и массы. Использование комбинированных полей с $p=-1$ для масс-спектрографов с двойной фокусировкой исследуется в [10].

Вывод: в силу принципа подобия траекторий однородные по Эйлеру электрические, магнитные и комбинированные поля могут быть основой для построения энерго- и масс-спектрографов с интересными аналитическими свойствами. Изложенные выше представления являются первым шагом к разработке техники синтеза таких спектрографов и хорошо согласуются с современными методами параллельного детектирования заряженных частиц. Высокий уровень современной технологии позволяет создавать электроды и магнитные полюса сложной трехмерной формы и делает эти идеи практически реализуемыми.

\section{Список литературы}

[1] Khursheed A. Scanning Electron Microscope Optics and Spectrometers. Singapore: World Scientific, 2010.

[2] Голиков Ю.К., Краснова Н.К. Теория синтеза электростатических энергоанализаторов. СПб.: Изд-во Политехн. ун-та, 2010. 409 с.

[3] Голиков Ю.К., Краснова Н.К. // ЖТФ. 2011. Т. 81. В. 2. С. 9-15.

[4] Краснова Н.К. // ЖТФ. 2011. Т. 81. В. 6. С. 97-103.

[5] Голиков Ю.К., Краснова Н.К. // Прикладная физика. 2007. № 2. С. 5-11.

[6] Голиков Ю.К., Краснова Н.К. // Научное приборостроение. 2014. Т. 24. № 1. C. $50-58$.

[7] Бердников А.С., Аверин И.А., Голиков Ю.К. // Масс-спектрометрия. 2015. T. 12. № 4. C. $272-281$.

[8] Бердников А.С., Аверин И.А., Голиков Ю.К. // Масс-спектрометрия. 2016. T. 13. № 1. C. 11-20.

[9] Бердников А.С., Аверин И.А. // Успехи прикладной физики. 2016. Т. 4. № 1. C. $89-95$.

[10] Бердников А.С., Аверин И.А. // Масс-спектрометрия. 2016. Т. 13. № 1. С. $62-$ 65.

Письма в ЖТФ, 2017, том 43, вып. 3 